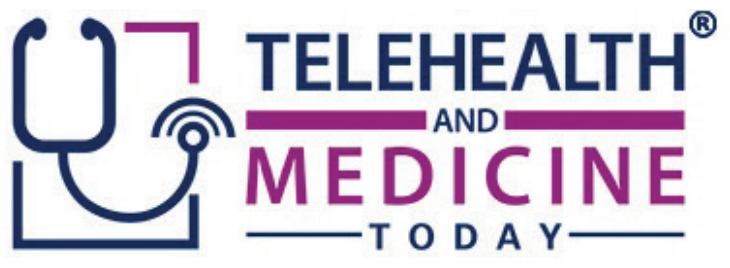

\title{
Improving Peritoneal Dialysis Access in Rural Communities with Telemedicine
}

\author{
Alexander Brauer, Sana Waheed
}

Affiliation: Division of Nephrology, Department of Medicine, University of Wisconsin School of Medicine and Public Health, Madison, WI, USA

Corresponding Author: Alexander Brauer, 5142 MFCB, 1685 Highland Avenue, Madison, WI 53705, USA. abrauer2@wisc.edu

Keywords: Hemodialysis, Nephrology, Peritoneal Dialysis, Public Health, Rural, Telehealth

Section: Research and Innovation

Increased availability of telehealth, along with patient satisfaction with the modality and financial incentives, makes peritoneal dialysis (PD) an excellent option for qualifying patients living in rural communities. Telemedicine has the potential to radically change the delivery of dialysis care by facilitating the ability of these patients to interact with their PD team via telehealth using a computer with reliable broadband Internet, which many rural clinics and hospitals now possess. Recent legal precedents make this a possibility. Therefore, it is recommended that renal providers take advantage of these favorable conditions to expand the diversity and quality of their practice.

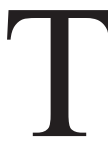
The main aim for the bundling policies implemented by the Centers for Medicare and Medicaid Services (CMS) in recent years was to lower utilization of separately billable medications, enhance quality of care, and promote access to dialysis services. ${ }^{1}$ It also seemed plausible that these changes would promote increased use of $\mathrm{PD}$, which costs less than hemodialysis (HD) for a dialysis center. ${ }^{2}$ Briefly, PD is a type of dialysis where blood filtration occurs at the peritoneal membrane in the abdomen, whereas in HD filtration occurs through an artificial filter in a machine. Despite mounting evidence that PD and HD are equivalent in terms of patient survival and quality of life, with higher treatment satisfaction in patients with $\mathrm{PD}$, the prevalent rates of $\mathrm{PD}$ remain stagnant nationally., The latest data trends available suggest that among people starting renal replacement therapy in the United States, $87.3 \%$ received HD, while $9.7 \%$ received $\mathrm{PD}$, with the rest receiving a preemptive kidney transplant. ${ }^{5}$ The use of PD has especially increased in urban markets, where access to PD is already abundant. ${ }^{6}$ This trend is most likely due to a combination of high concentration of 
dialysis facilities and patients in urban areas associated with utilization of PD based largely on whether a specific healthcare system is comfortable delivering this modality. ${ }^{7,8}$ As a result, the main increase in PD in recent years is found in urban areas adjacent to larger, often academic, medical centers. This is noteworthy because unlike HD, PD is a dialysis modality that is done in the patient's home and therefore prevents frequent travel to a HD clinic.

Many of the patients who would benefit directly from improved access to PD are located in rural areas where issues of transportation and access to care are fundamental. We argue that patients from rural areas are the most likely to benefit from expansion of $\mathrm{PD}$, and recent developments in healthcare delivery, such as telehealth, can bolster these efforts.

\section{RURAL POPULATIONS AND \\ DIALYSIS ACCESS IN WISCONSIN}

Patients on dialysis who live in isolated areas ( $>18$ miles from treatment facility) have an increased mortality rate compared with those living closer. ${ }^{9}$ The issue of access to healthcare is recognized and the CMS published last year a
"Rural Health Strategy" in an attempt to generate renewed attention to the unique needs of these communities. ${ }^{10}$

To understand the disparities in access to PD and $\mathrm{HD}$, we mapped the location of every dialysis center in Wisconsin. We then cross-referenced data from the "Dialysis Facility Compare" tool from the Medicare.gov website, the "Dialysis Finder" tool developed by DaVita and the websites of individual dialysis facilities. An interactive map with comprehensive information regarding every dialysis facility charted can be found at https://maphub.net/abrauer2/Dialysis.

Wisconsin is an excellent case study because of its geographical diversity: extremely large metropolitan areas in the southeast contrast the remote rural regions in the Southwest and Northern part of the state. Centers offering PD are almost nonexistent in rural areas of Wisconsin compared to HD centers (Figure 1). The trend mentioned earlier that PD expanded mostly in urban areas in the last decade seems to be validated by this mapping effort. There is no reason to believe similar trends would not be found across the United States.

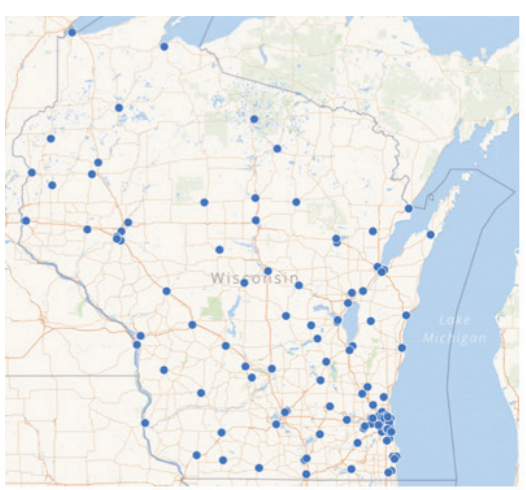

A.

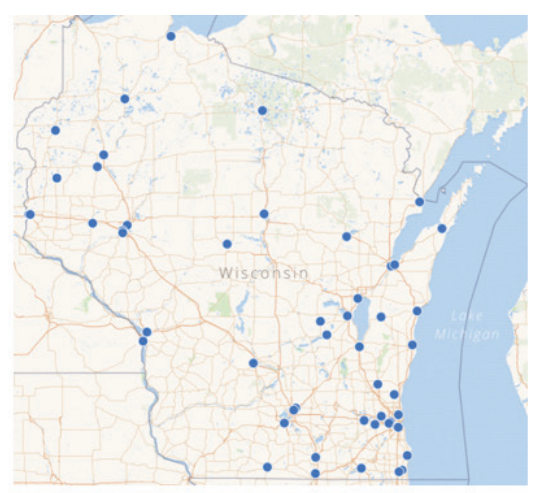

B.

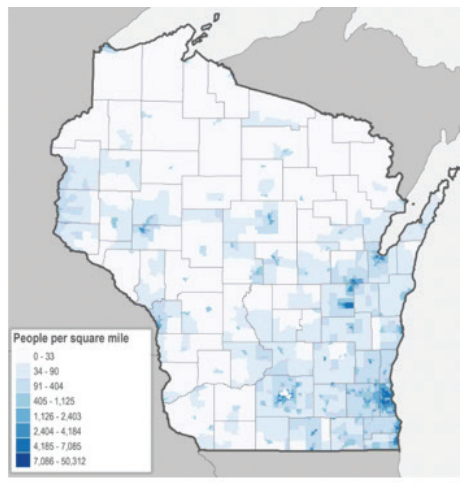

C.

Figure 1-(A) Map of Wisconsin with dots representing a center offering hemodialysis services. (B) Map of Wisconsin with dots representing a center offering peritoneal dialysis services. (C) Map of Wisconsin depicting population density, with higher densities represented in dark blue.

Source: MapHub, Caitlin McKown/UW Applied Population Laboratory. https://www.wiscontext.org/ putting-rural-wisconsin-map 
Even considering that patients on PD only have to visit their clinic once per month, distance can still be a major factor. For example, a patient on dialysis living in Richland Center, a city located in the middle of the rural Southwestern area, would have to travel 2 hours or more to have an appointment at a dialysis center that has staff trained in PD administration. It might be more convenient for that patient to drive to the outskirts of the town for HD 3 times a week. Perception of convenience and provider comfort with HD for patients located in rural communities directly or indirectly shapes their modality choice.

This is not to say that PD is suited for everyone living in rural areas - it definitely is not. Many patients have clinical profiles that make them much more suited for HD and having centers that offer this modality in rural areas is a strength that should be recognized. At the same time, not offering PD to rural patients that might clearly benefit from it is a shortcoming that can (and should) be addressed by renal healthcare providers.

\section{TELEMEDICINE AS A TOOL TO INCREASE PD AVAILABILITY IN RURAL AREAS}

Telemedicine has the potential to radically change the delivery of dialysis care. Currently, a factor preventing many patients from choosing PD (assuming they are eligible) is the long driving distances to a center that is comfortable with this modality. If a patient was able to interact with their PD team via telehealth, they could potentially live hours away as long as they have reliable access to a computer with broadband Internet, which many rural clinics and hospitals now possess. Recent legal changes have made this a possibility. The Bipartisan Budget Act of 2018 explicitly states that patients receiving dialysis at home (such as PD) "may choose to receive monthly end-stage renal disease-related clinical assessments furnished on or after January 1, 2019 via telehealth." ${ }^{11}$ Under the law, patients would still be required to have face-to-face appointments monthly for the first 3 months and every 3 months thereafter. In other words, the number of in-person visits after the first 3 months on PD would be cut down from 12 to 3 and replaced with nine telehealth visits. There would be no change in the payment to the physician or the dialysis facility if a patient was to switch to telehealth.

It quickly becomes apparent that for eligible patients, the use of telehealth can transform PD into a desirable option for those living in isolated areas. Furthermore, it does not require opening new PD centers but rather using remote technology available at many rural hospitals and clinics. Rather than spending money on creating new centers or programs, this approach encourages a shift in our current dialysis care model. The emphasis is transferred from the dialysis center and to communication between patients and providers.

\section{CONCLUSION}

Increased availability of telehealth, financial incentives, and, most importantly, patient satisfaction with the modality make PD an excellent option for patients living in rural communities. Renal providers should take advantage of these favorable conditions to expand the diversity and quality of their practice.

Conflicts of Interest: The authors have no conflicts of interest to disclose.

Funding statement: None

Contributors: Both authors contributed to the development of the article and subsequent editing. 


\section{REFERENCES}

1. Erickson KF, Winkelmayer WC. Evaluating the evidence behind policy mandates in US dialysis care. JASN. 2018;29(12):2777-9. https://doi.org/10.1681/ASN.2018090905

2. Golper TA. The possible impact of the US prospective payment system ("Bundle") on the growth of peritoneal dialysis. Perit Dial Int. 2013;33(6):596-9. https://doi.org/ 10.3747/pdi.2013.00212

3. Wong B, Ravani P, Oliver MJ, et al. Comparison of patient survival between hemodialysis and peritoneal dialysis among patients eligible for both modalities. Am J Kidney Dis. 2018;71(3):344-51. https://doi. org/10.1053/j.ajkd.2017.08.028

4. Iyasere OU, Brown EA, Johansson L, et al. Quality of life and physical function in older patients on dialysis: A comparison of assisted peritoneal dialysis with hemodialysis. CJASN. 2016;11(3):423-30. https://doi.org/10.2215/CJN.01050115

5. United States Renal Data System. 2016 USRDS annual data report: Epidemiology of kidney disease in the United States. Bethesda, MD: National Institutes of Health, National Institute of Diabetes and Digestive and Kidney Diseases; 2016 [cited 2017 Aug 3]. Available from: https://www. usrds.org/2016/view/Default.aspx

6. Sloan C, Coffman C, Sanders LL, et al. Impact of Medicare dialysis bundled payment reform on availability of peritoneal dialysis in United States. J Gen Intern Med. 2018;33:S227.

7. Kaplan AA. Peritoneal dialysis or hemodialysis: Present and future trends in the United States. Contrib Nephrol. 2017;189:61-4. https://doi.org/ 10.1159/000450672

8. Wang V, Maciejewski ML, Coffman CJ, et al. Impacts of geographic distance on peritoneal dialysis utilization: Refining models of treatment selection. Health Serv Res. 2017;52(1):35-55. https://doi.org/ 10.1111/1475-6773.12489

9. Ajmal F, Bennett KJ, Probst JC. Geographic disparities in mortality among the end stage renal disease patients: An analysis of the United States Renal Data System, 2007-08. J Nephrol. 2016;29(6):817-26. https://doi. org/10.1007/s40620-016-0324-3

10. CMS Rural Health Strategy | CMS. May 2018 [cited 2019 Mar 11]. Available from: https://www.cms.gov/newsroom/fact-sheets/ cms-rural-health-strategy

11. Bieber SD, Weiner DE. Telehealth and home dialysis: A new option for patients in the United States. CJASN. 2018;13(8):1288-90. https://doi. org/10.2215/CJN.03010318

Copyright Ownership: This is an open access article distributed in accordance with the Creative Commons Attribution Non Commercial (CC BY-NC 4.0) license, which permits others to distribute, adapt, enhance this work non-commercially, and license their derivative works on different terms, provided the original work is properly cited and the use is noncommercial. See: http://creativecommons. org/licenses/by-nc/4.0. 